Document downloaded from:

http://hdl.handle.net/10251/170288

This paper must be cited as:

Fico, G.; Martinez-Millana, A.; Leuteritz, J.; Fioravanti, A.; Beltrán-Jaunsarás, ME.; Traver Salcedo, V.; Arredondo, MT. (2019). User Centered Design to Improve Information Exchange in Diabetes

Care Through eHealth: Results from a Small Scale Exploratory Study. Journal of Medical Systems. 44(1):1-12. https://doi.org/10.1007/s10916-019-1472-5

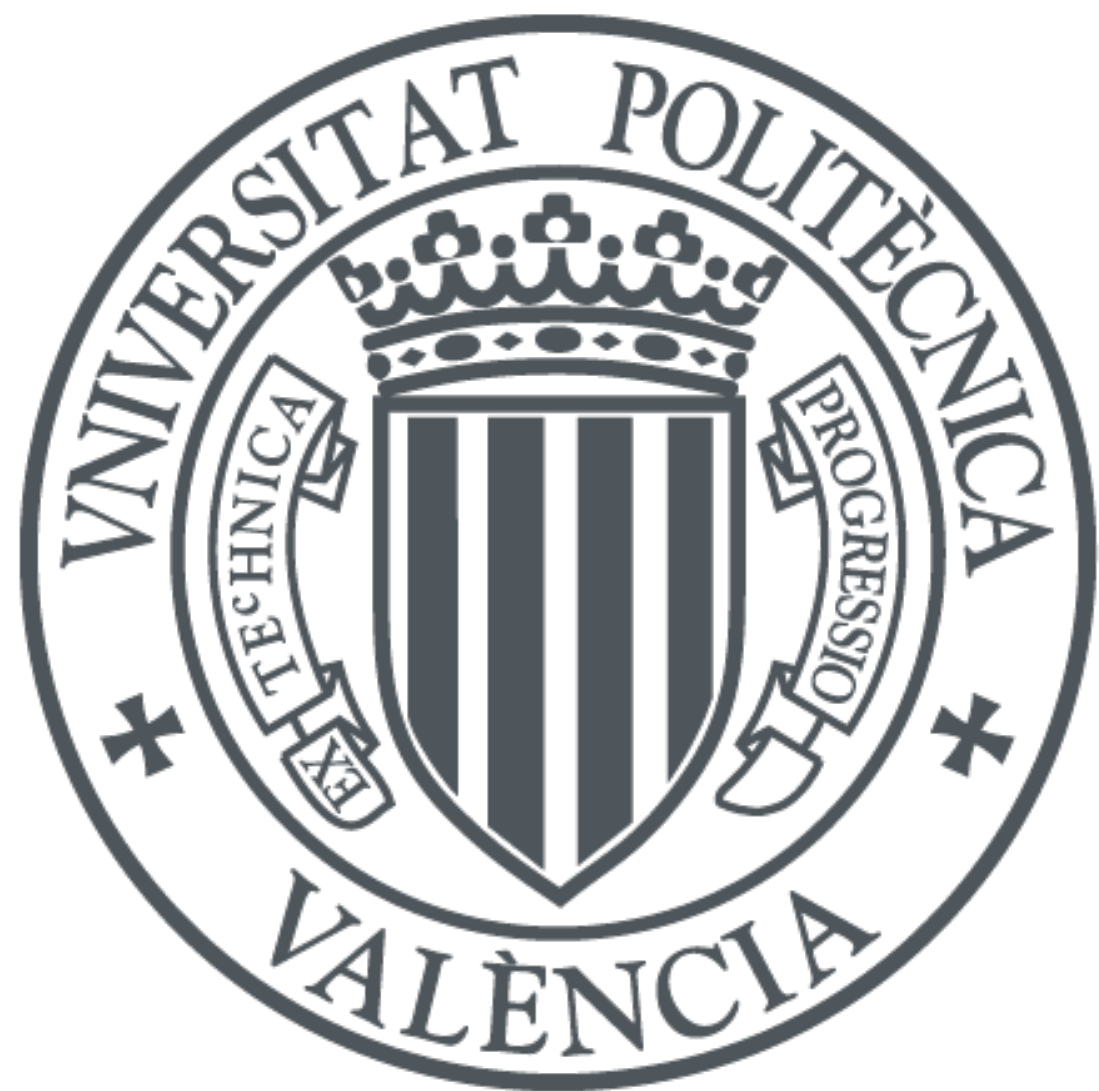

The final publication is available at

https://doi.org/10.1007/s10916-019-1472-5

Copyright Springer-Verlag

Additional Information 
Noname manuscript No.

(will be inserted by the editor)

\title{
User Centered Design to improve information exchange in Diabetes Care through eHealth
}

\section{Results from a small scale exploratory study}

\author{
Giuseppe Fico • Antonio \\ Martinez-Millana • Jan-Paul Leuteritz • \\ Alessio Fioravanti · Maria Eugenia \\ Beltran • Vicente Traver - Maria Teresa \\ Arredondo
}

Received: date / Accepted: date

\begin{abstract}
Heterogeneity of people with diabetes makes maintaining blood glucose control and achieving therapy adherence a challenge. It is fundamental that patients get actively involved in the management of the disease in their living environments. The objective of this paper is to present and evaluate the processes needed to build a supporting self-management system for diabetes in community settings. We used User Centered Design techniques for defining and personalizing an eHealth solution for the management of diabetes, and assessing the use and compliance of a self-management system in a multicenter randomized pilot study. Particularly, User Centered Design principles were used to involve diabetic patients and treating professionals into the design, development and evaluation of a self-management system; which comprised three iterative cycles: scenario definition, user archetype definition and system development. A comprehensive system was developed integrating modules for the management of blood glucose levels, medication, food intake habits, physical activity, diabetes education and sending messages. The sys-
\end{abstract}

G. Fico, A. Fioravanti, M.E. Beltran and M.T. Arredondo

Universidad Politcnica de Madrid

Photonics Technology and Bioengineering Department

Life Supporting Technologies Group, Madrid, Spain.

E-mail: gfico@lst.tfo.upm.es

A. Martinez-Millana, V. Traver

ITACA.Universitat Politècnica de València, Valencia, Spain

J.P. Leuteritz

Ergonomics and Vehicle Interaction

Fraunhofer-Institute for Industrial Engineering (IAO), Berlin, Germany

Funding Statement

European Commission under the 7th Framework Program grant agreement number 216270.3 . 
tem was adapted for two types of principal users (personas): Type 1 Diabetes user and Type 2 Diabetes user. 20 patients and 24 treating professionals were enrolled in the study and used the system for a period of four weeks. The assessment of usage and compliance metrics yielded similar results, which differed significantly among the two types of users, except for the medication module, which showed a significantly different use and compliance $(p=0.01)$.

Keywords Personal Health Records · User Centered Design · Diabetes Management $\cdot$ mHealth $\cdot$ co-design

\section{Introduction}

Modern health care systems are designed to treat acute diseases rather than managing chronic conditions [1]. Chronic diseases would require long-term care management programs to first stabilize patients and then prevent or delay complications [2]. Diabetes Mellitus is a paradigmatic case of long-term care, being one of the most prevalent diseases worldwide, with more than 380 million patients with no cure [3].

The most common forms of diabetes are Type 1 Diabetes Mellitus (T1DM) and Type 2 Diabetes Mellitus (T2DM). An impaired insulin secretion causes T1DM, which leads to chronic hyperglycemia and accounts for $10 \%$ of all diabetes cases. T2DM is caused by an inadequate insulin secretion and an impaired insulin action, and accounts for almost $90 \%$ of all diabetes cases. Generally, T1DM is caused by an autoimmune reaction in individuals under 20 years of age, while T2DM is associated with aging, lifestyle and genetic predisposition among individuals over the age of 50 . The clinical heterogeneity of these patients challenges the process of care, which is focused on maintaining blood glucose control [4]. It is paramount to involve both types of patients in the management of the disease inside their living environments. This requires taking medicines, following a proper diet, doing physical exercise and being informed and trained about self-management and decision making. Moreover, patients should be supported and followed-up by practice teams who check adherence to care plans, perform therapy readjustment and mitigate the risk of complications[4].

In this context, the Information Technology (IT) applications have contributed to perform an efficient and personalized follow-up of the disease [5]. These systems allow monitoring multi-parametric data and performing analysis of relevant parameters such as physiological measurements, laboratory examinations, and lifestyle data, thus enabling more precise follow-up of patients through better quality and more comprehensive interpretation of data and delivery of alerts, warnings, and support to decision making [5]. In order to achieve this, the main challenge is to understand how to manage data heterogeneity and train patients to successfully use such technology.

State of the art systems include new ways to register daily-based events such as the quantification of the ingested meal contents in carbohydrates, proteins and fats, based on a picture of the meal to decompose the nutritional 
value and calculate the insulin dose $[6,7]$. Other types of systems try to integrate real-time continuous glucose sensors, insulin pumps and a mobile-based tele-medicine system to create a closed-loop of communications with the doctor $[8,9]$.

Electronic Health Records (EHR) [10, 11], Personal Health Records (PHR)[12], mHealth [13] and eHealth [14] shall improve the prevention and treatment at the point of care through remote therapeutic decision-making and follow-up relying on adherence to care plan and towards data insertion through apps and sensors. These types of technologies have been assessed in several studies, focused on defining strategies to collect Observations on Daily Living (ODL) to support behavioural monitoring [15], exploring how ODL can be displayed to users in a meaningful manner[16, 17].

As regards platforms for the self-management of diabetes, one of the most used systems is Bluestar by WellDoc [18], which allows patients to track and record blood sugar levels offering a real-time response and clinical basis. In 2011, the Salzburg statement confirmed the potential of Decision Support Systems (DSS) in the shared decision-making and self-management of diseases $[19,20]$. But, how patients and health professionals can use these technologies in the long term remains an unanswered question [21]. Patients need to be educated about the role they play in the process of care, the information they need to manage, and the criteria for selecting tools that would help them understanding the consequences of their decisions[22].

The work reported in this paper describes the design and implementation of an application system for diabetes management integrating User Centred Design (UCD) principles into the development cycle. Our hypothesis is that UCD techniques allow identifying the most important elements for self-monitoring of the diabetes disease and, in turn, creating solutions that can support effective, sustainable and useful adoption of PHRs. The application system was tested in a small-scale pilot including 20 T1DM and T2DM patients for a period of four weeks. Usage and compliance of the co-designed system show a significantly improved behaviour of patients towards the applications for each of the modules, which enabled tailored decision-making actions.

\section{Methods}

2.1 Extraction of user requirements and system definition

The complexity of diabetes mechanisms does not allow defining a single diabetic patient profile and, in turn, a single scope for a disease management system. It is under this assumption that the European research project METABO started its activities: a consortium composed of biomedical and information technology researchers, health technology industries, clinical engineers, HCI experts, and research hospitals worked more than four years on the definition, implementation and validation of a diabetes disease management platform[23]. UCD is a design methodology that aims at involving users in the design, de- 
velopment and evaluation of systems and products[24, 25]. The methodology of this study consisted in three iterative cycles: usage scenario definition, user archetype definition and system development, as described in Fig. 1. UCD provides a considerable amount of upfront research and analysis prior to development.

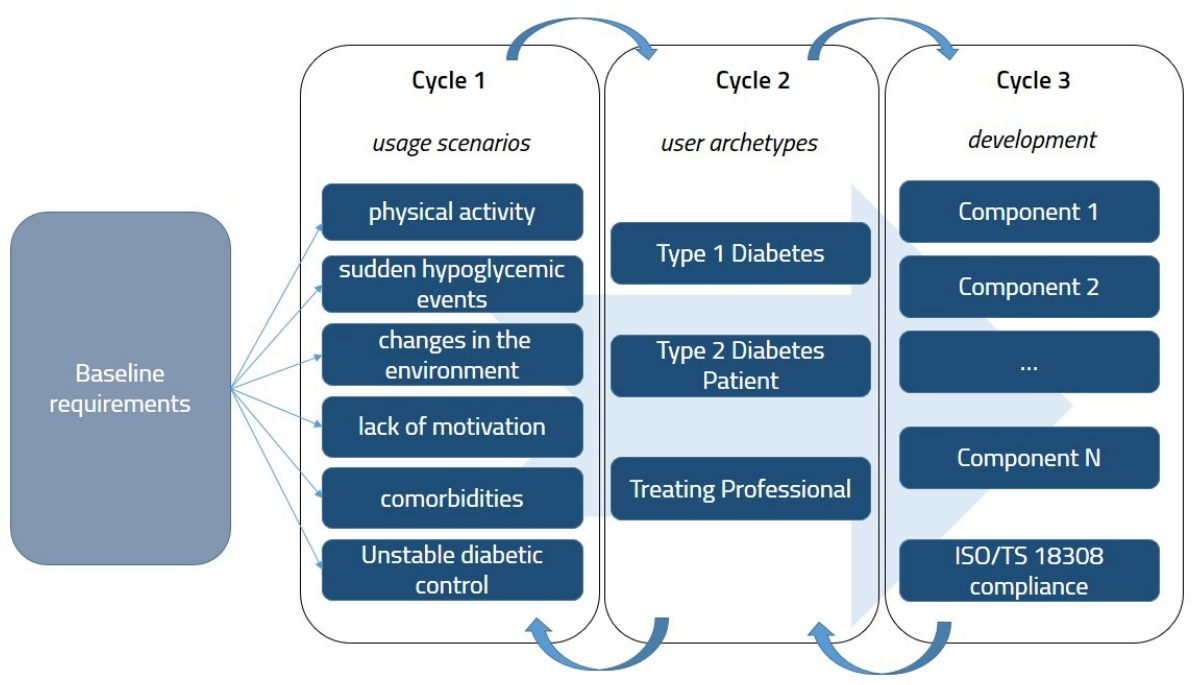

Fig. 1 Implementation of the UCD methodology in 3 cycles

In the first cycle we defined a situation/problem-oriented representation of the disease involving patients and clinicians. Scenarios were based on international standards of care (by the National Health System in the UK [26] and the American Diabetes Association [3]) and six specific needs: sudden hypoglycemic events, changes in the environments, physical activity, lack of motivation, co-morbidities, unstable diabetes control[27]. The second cycle involved defining technological factors to support these scenarios. We identified four type of form factors: 1) sensors to allow physiological monitoring of blood glucose, physical activity and weight, 2) tools for collecting ODL and integrating the sensed data from the patient side, 3) tools for treatment and follow up from the practice team side, and 4) a system to allow exchange of data and establish a communication channel between patients and careers. These factors were used for the creation of mock-ups that were evaluated and refined through focus groups and face-to-face interviews with end-users. Results were used for the definition of three main user archetypes, called PRIMARY PERSONAs according to UCD theory: T1DM patient, T2DM patient, and the Treating Professional (TP). A heuristic assessment of the mock-ups according to Nielsens gold standard 10 Usability Heuristics for User Interface Design [25] and the definition of scenarios based on the PERSONAs, led to the development of the first version of the prototypes (second cycle). The prototypes 
were assessed in usability tests and interviews with patients representing the different PERSONAs, providing feedback and substantial changes in the final system development (third and final cycle).

\subsection{Study description and data analysis}

The system was tested in a small-scale exploratory pilot study in four clinical centers in Modena, Parma, Prague and Madrid from January 2015 to September 2015. The Medical Ethics Committee of each center approved the study protocol, and all patients gave their informed consent in order to be enrolled in the study. We recorded usage and compliance metrics of a group composed by 13 T1DM and 7 T2DM patients who used the PMDs during four consecutive weeks (Table 1). A total of 24 care providers used the Control Panel (CP), a desktop-based application designed for the treating professionals. However, in each of the four centers there were one or two diabetes specialists assigned as the main user of the CP. These specialists were assisted by other doctors, nutritionists and nurses.

The applications were designed to record all the interactions of the users with the system. To assess user behaviour, the number of accesses to each module was recorded together with a timestamp. Moreover, to evaluate the communication performance, the number of messages from each module (i.e. packages) was also recorded. A package is the simplest representation of a transaction of information regarding a specific module. For instance, an insulin intake package would contain information about the type of insulin and pumped dose. Records were compiled and analysed independently for T1DM and T2DM PERSONAs. Due to the non-parametric distribution of the observed variables, a Wilcoxon independence test was calculated to find out if the behaviour on the access to modules and communications had differences not attributable to chances. The Wilcoxon test was chosen as a particularly conservative method, sacrificing test-power for accurateness under possibly non-parametric conditions, and significance level was accepted for $\mathrm{p}$ values under 0.05 at $95 \%$ confidence interval.

Moreover, two more indicators related to the user response to technology and treatments were defined. First we calculated the Usage parameter in Equation (1) which gives an approximation about the intensity of the subject's use of the system. It relates the number of days that a subject has to perform an action (A) (monitoring blood glucose, insulin administration, eating a specific meal, etc.) and the number of prescribed actions not reported by the subject (B). Second, we measured the level of Compliance in Equation (2) by dividing the number of recorded events $(R)$ by the number of prescribed events $(p)$.

$$
\begin{gathered}
\text { Usage }=(1-B / A) \% \\
\text { Compliance }=(R / p) \%
\end{gathered}
$$




\begin{tabular}{|c|c|c|c|}
\hline & T1DM $(\mathrm{n}=13)$ & T2DM $(\mathrm{n}=7)$ & $p$-value \\
\hline Characteristic & n (\%) & n (\%) & \\
\hline Gender & & & 0.68 \\
\hline Male & $9(69 \%)$ & $5(71 \%)$ & \\
\hline Female & $4(31 \%)$ & $2(29 \%)$ & \\
\hline Studies & & & 0.21 \\
\hline Undergraduate & $2(15 \%)$ & $2(29 \%)$ & \\
\hline Secondary & $3(23 \%)$ & $2(29 \%)$ & \\
\hline University & $8(61 \%)$ & $3(43 \%)$ & \\
\hline $\mathrm{PhD}$ & $0(0 \%)$ & $0(0 \%)$ & \\
\hline Marital Status & & & 0.40 \\
\hline Single & $7(54 \%)$ & $2(29 \%)$ & \\
\hline Married & $5(38 \%)$ & $5(71 \%)$ & \\
\hline Divorced & $1(8 \%)$ & $0(0 \%)$ & \\
\hline Widowed & $0(0 \%)$ & $0(0 \%)$ & \\
\hline Medical Variables & $m \pm s d$ & $m \pm s d$ & \\
\hline Age (years) & $38.2 \pm 10.6$ & $48.3 \pm 11.3$ & 0.06 \\
\hline Duration of Diabetes (years) & $15.5 \pm 10.6$ & $9.1 \pm 6.7$ & 0.17 \\
\hline BMI & $24.3 \pm 3.6$ & $31.3 \pm 3.8$ & $<0.001$ \\
\hline Waist/Hip & $84.7 \pm 15.2$ & $109.5 \pm 13.5$ & 0.02 \\
\hline Hb1Ac & $7.6 \pm 1.3$ & $7.8 \pm 1.4$ & 0.50 \\
\hline FPG & $179.6 \pm 70.8$ & $144.7 \pm 46.1$ & 0.10 \\
\hline \multicolumn{4}{|l|}{ Lifestyle } \\
\hline Smoking & $2(15 \%)$ & $2(33 \%)$ & 0.91 \\
\hline Alcohol & $4(31 \%)$ & $3(50 \%)$ & 0.96 \\
\hline Limitations on Diet & $1(8 \%)$ & $1(17 \%)$ & 0.75 \\
\hline \multicolumn{4}{|l|}{ Physical Activity } \\
\hline Days per Week & $3.3 \pm 2.7$ & $4.3 \pm 3.3$ & 0.46 \\
\hline Duration (min) & $41.5 \pm 43.3$ & $36.4 \pm 32.2$ & 0.87 \\
\hline Intensity (METs) & $2.9 \pm 2.6$ & $2.7 \pm 2.3$ & 0.79 \\
\hline
\end{tabular}

Table 1 Demographic description of the study sample grouped by the PERSONA TYPE and homogeneity test

The pilot study aimed at assessing the acceptance of the system in a real world context. This was achieved by analysing the user satisfaction and the usefulness perceived by its users and the user behaviour at the end of the pilots:

- User satisfaction: we used the AttrakDiff questionnaire [28]. The AttrakDiff is consists of 4 subordinate constructs, all of which are computed separately: pragmatic quality, the two hedonic qualities stimulation and identification, and attractiveness. Whereas pragmatic quality might be considered to be the best representation of user satisfaction, a product such as METABO should neither disregard the other dimensions, which are rather connected to the concept of user experience. Our quality criterion consisted in having the confidence interval of the collected measures mean value over a score of 3 .

- Perceived usefulness: we constructed a questionnaire for assessing the usefulness measure based on Davis questionnaire [29] and some additional custom items. Cronbachs alpha was used to check results homogeneity. 
The arithmetic mean value of all the remaining items was calculated and the quality criterion consisted in finding the scales middle score outside the confidence interval of the collected measures mean value.

\section{Results}

\subsection{Personas and Scenarios}

The most frequent tasks that T1DM people perform are related to the daily annotation of medical and lifestyle data. The user needs to understand in real time how to deal with the multiple factors affecting blood glucose levels, without compromising their quality of life. Users demanded features to resemble as much as possible the actions they usually perform, for instance filling in paper diaries or notebooks where patients record their measurements and add comments. Data reviewing was spotted as more time-consuming and less suitable for doing on the move. However, it is an action that T1DM people may wish to perform, possibly comparing different sources of information to understand what is compromising an optimal glucose control (typically glucose vs. food intake vs. insulin injections).

In the case of T2DM, the main objective was on diabetes education, communication with health care professionals and empowerment to adopt a healthier lifestyle. TD2M people will usually not be insulin dependent, but likely to suffer from a number of co-morbidities, and probably have a complex medication regime. Empowering patients to change their routines and to adopt a healthier lifestyle entails the provision of educational and motivational materials in combination with a behaviour change strategy. Although an interface for recording measurements should still be present in the application, lifestyle management and communications with health professionals should be considered as a key factor.

The Treating Professional (TP) is a diabetologist, an endocrinologist, or a case manager who typically treats many patients per day (about 30 per day, as extracted from interviews) and has 5-10 minutes for each of them. In this time frame, they need to understand the patient profile, the clinical status, as well as the current treatment and, based on this, take decisions regarding changes in the treatment and respective counselling. In addition, nurses, educators, and nutritionists also participated in this provision of care schema. They need access to the same kind of information but not to the entire set and not to all the features with respect to the TP (they are defined as SECONDARY PERSONA).

\subsection{The METABO Platform}

The METABO platform (Fig. 2) is a system developed on the basis of the requirements of the two first iterative cycles. METABO is conceived by patients as an electronic diary, equipped with some intelligence which supports 
them during their daily activities. This intelligence supported them in what to do and when to do it, based on the treatment they agreed on with the doctor, helping them to properly visualize their record and allowing them to exchange messages with their caregivers. The modules, and consequently the information categories in METABO were Food Intake (FI), Blood Glucose (BG), Drug Intakes (MD), Physical Activity (PA), Education Module (ED), and a Messaging module for the management of feedback from both the system and doctor (MS). Data gathered and managed by the different modules were consumed by the different services (feedback, data synchronization and filtering, authentication and security mechanisms, etc.) running locally in the applications and managed by a Central Server (CS). Patient interfaces, named Patient Monitoring Devices (PMD), consisted of applications running in Smartphones (HTC HD2 for T1DM and iPhone 4.0 for T2DM) and desktop PCs (Asus Tablet PC, named Patient Panel, for both patient PERSONAs). Smartphone applications were designed to provide better support for data insertion activities, especially in mobility environments, while the Patient Panel was conceived for sensor download and content visualization activities. The Control Panel (CP)provided to the TP was a java client for desktop PCs. The Central Server (CS) was installed on a host machine, managing the PMDs and the CPs installed in all the centers, and executing registration, security, authentication, data storage, synchronization, interoperability, scheduling, and feedback services. Four type of sensors were provided to the patients (Fig. 2 orange rectangle). T1DM patients were equipped with a Continuous Glucose Monitoring System and an activity holter. T2DM patients were equipped with a strip blood glucose sensor and a pedometer. Further details on the type of sensors and integration can be found in [9]. The system included an automatic delivery of feedback messages based on a database of predefined actions and rule engine, which evaluated a set of metrics related to the compliance of the patient and provided customized motivational messages. This automatic feedback component has been previously presented by co-authors [30]. The main purpose of this smart feedback mechanism was to let patients not only record data, but actively use and learn from it (which is the intended benefit of a PHR) and access information that may help them take better decisions.

The overall usage scenario was: 1) Treating Professional (TP) launches the $\mathrm{CP}$ and creates a new instance for a patient, inserting clinical profile and lifestyle scenario. 2) TP makes the prescription in terms of what the patient needs to annotate (FI, BG, PA, ED, MS), at which frequency (measurements per day, per working day vs. weekend or per week) and sets up thresholds where needed. 3) The Central Server (CS) transforms the prescription into rules for the creation and the configuration of the PMDs: modules were enabled or disabled, and parameters were transformed into rules for the feedback services, analysing insertion frequency and adherence. 4) Patient is registered into the system and is given the PMDs, and leaves after a training phase .5) Patient status and treatment adherence are periodically checked by the CS, and feedback messages are delivered. 6) As soon as the TP opens the CP, she could check which patients need to be analysed, based on number and 


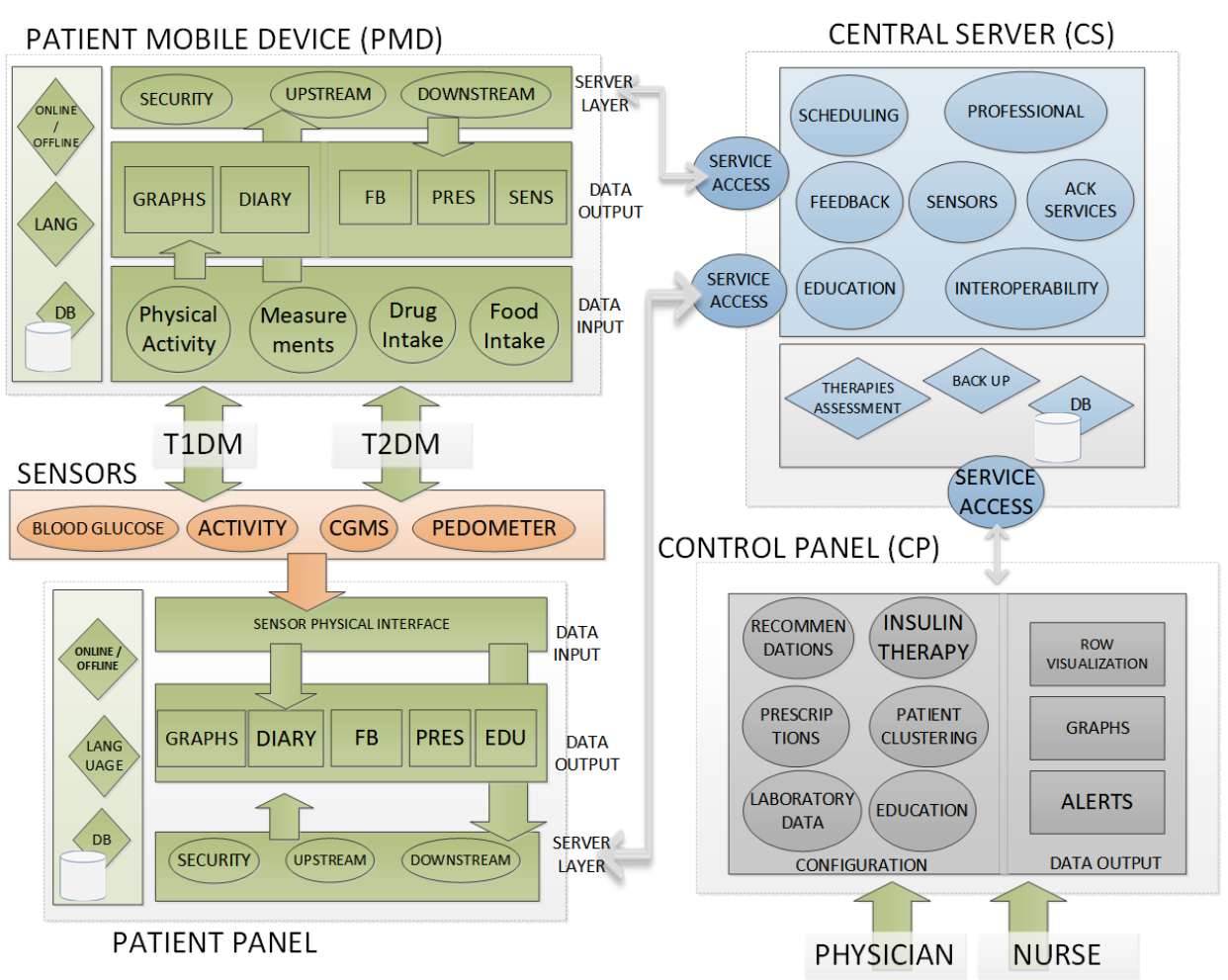

Fig. 2 System architecture

priorities of specified alerts, as well as according to the scheduled visits and unread messages. Such alerts were configurable by the TP and related to the metabolic control of the disease and the compliance to the prescriptions, e.g., Number of blood glucose tests per day, Number of blood glucose excursions over a threshold, Time devoted performing physical activity, and Self-reporting of meals and insulin intake (Fig. 3).

\subsection{System evaluation}

We measured how patients used the modules during the entire duration of the study. Number of accesses to each module (sessions) and number of records sent from each module (data packets) were recorded automatically. According to the aggregated analysis (Fig. 4 and Fig. 5), the most frequently used modules were those for Food Intake and for Medication. With respect to the overall access to the four modules, T1DM average usage decreased from more than 70 sessions during Week 1 to 50 sessions in week 3 and less than 10 during the last week. While the first decrease can be positively associated with the learning 

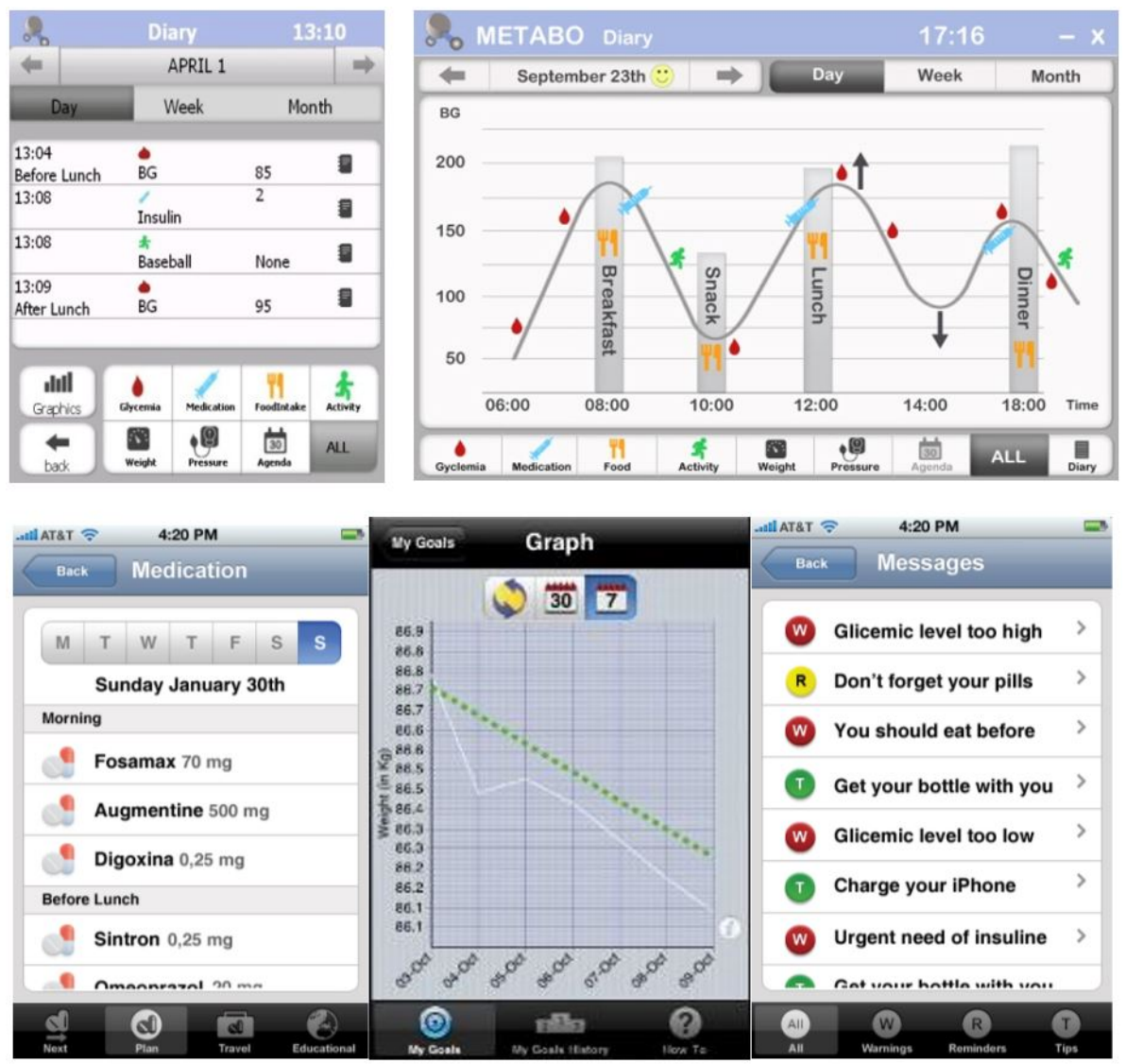

Fig. 3 T1DM Graphics (up); T2DM Drug Intake, Goal Achievements and feedback modules (bottom)

phase on the usage of the system, low values in the last week may be related with the fact that T1DM were also sending sensor measurements through the continuous glucose monitoring and physical activity sensors, without the needs of accessing to these modules. This was not observed for T2DM, where the usage was acceptable in all modules until the end of the trial. Table 2 shows the results of the behaviour analysis based on Wilcoxon independence test for the use and Table 3 for the communication of the modules. This test has also been applied to the average indicators for the entire study duration (Table 4), confirming that the Medication module has a different communicating pattern among the two type of users, whereas, the rest of the modules have a similar behaviour in the use and the communication.

In both cases, the prescribed usage (how many times the patients uploaded information compared to how often they were expected to do so) and compliance (how well the patients achieved what was prescribed) are above thresholds 

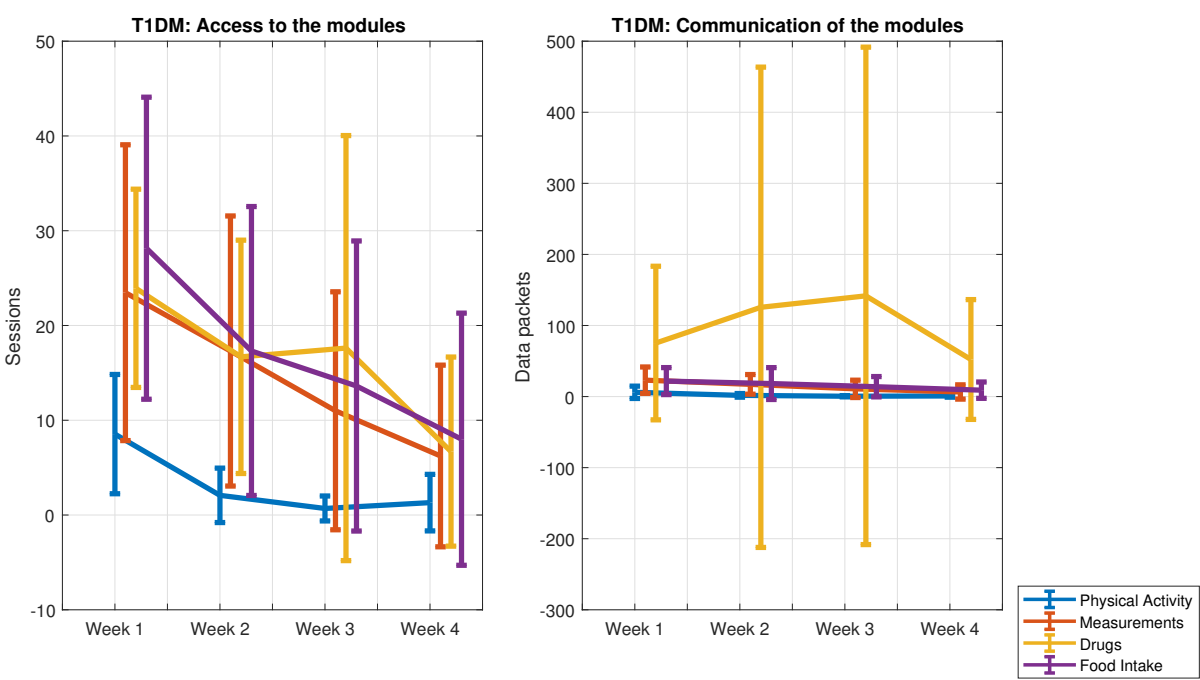

Fig. 4 Evolution on the average use (number of sessions) and average communication (number of packets) for each of the modules in the applications for T1DM persona for each week.
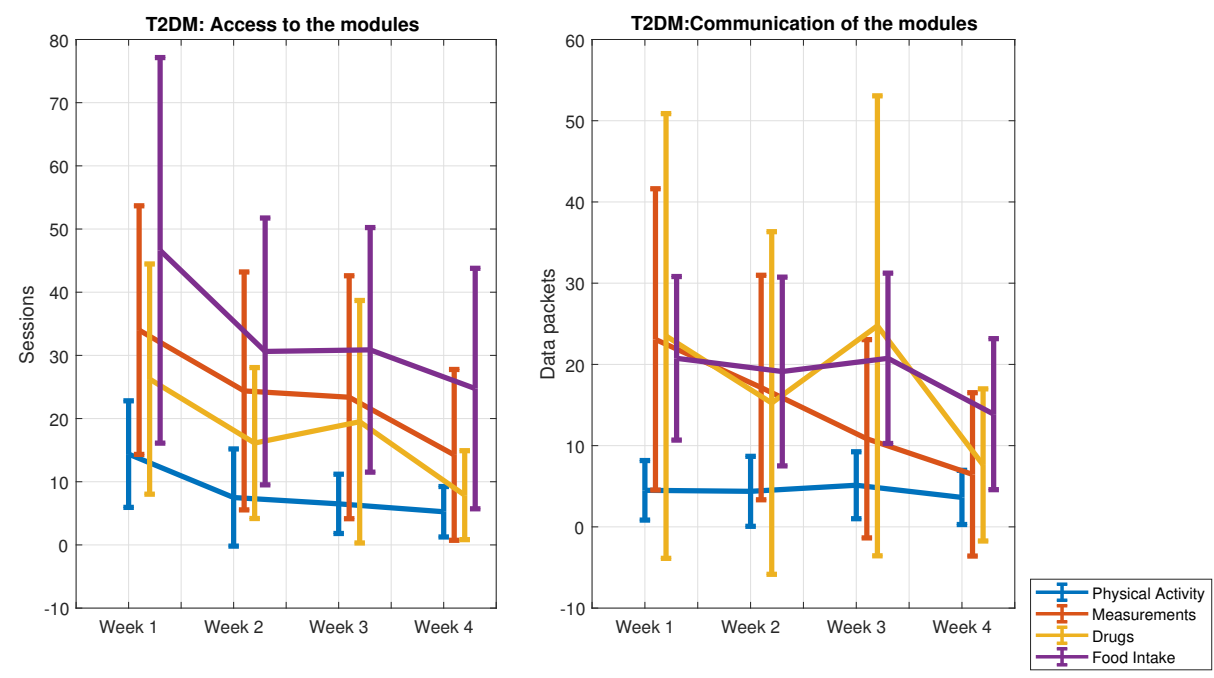

Fig. 5 Evolution on the average use (number of sessions) and average communication (number of packets) for each of the modules in the applications for T2DM persona for each week

that were considered as acceptable by the clinical experts and did not decrease substantially during the study.

A more active role was observed in T2DM patients on the usage of the educational functionalities with respect to T1DM (70\% out of the total number of patients using the education modules were T2DM). As regards the messages 


\begin{tabular}{lcccc}
\hline MODULE & Week 1 & Week 2 & Week 3 & Week 4 \\
\hline Physical Act & .15 & .16 & $<.001$ & $\mathbf{. 0 2}$ \\
\hline Measurements & .19 & .40 & .09 & $\mathbf{. 0 4}$ \\
\hline Drugs & .94 & .71 & .51 & .32 \\
\hline Food Intake & .14 & .15 & $\mathbf{. 0 4}$ & $\mathbf{. 0 2}$
\end{tabular}

Table 2 Wilcoxon independence test $\mathrm{P}$ values for T1DM/T2DM for use

\begin{tabular}{lcccc}
\hline MODULE & Week 1 & Week 2 & Week 3 & Week 4 \\
\hline Physical Act & .77 & .19 & $\mathbf{< . 0 0 1}$ & $\mathbf{. 0 2}$ \\
\hline Measurements & .51 & .80 & .21 & .12 \\
\hline Drugs & .15 & .39 & .49 & .48 \\
\hline Food Intake & .91 & .64 & .19 & .13
\end{tabular}

Table 3 Wilcoxon independence test $\mathrm{P}$ values for T1DM/T2DM for communication

\begin{tabular}{lcc}
\hline MODULE & ACCESS & COMMUNICATION \\
\hline Physical Act & .20 & .34 \\
\hline Measurements & .20 & .99 \\
\hline Drugs & .89 & $\mathbf{. 0 3}$ \\
\hline Food Intake & .06 & .43
\end{tabular}

Table 4 Wilcoxon independence test of overall records in access and communication of the modules among T1DM and T2DM users

with the TP, T1DM exchanged an average of $4.9 \pm 3.9$ messages, while T2DM an average of $6.6 \pm 3.0$. The messages sent by the system (reminders, alerts, tips and recommendations) where $124.5 \pm 52.4$ for T1DM and $188.3 \pm 57.4$ for T2DM. This also shows a difference between the two type of patients, being T2DM more focused on education activities.

The compliance value represents the fit between the measurement values introduced by the patients and their prescription (Fig. 6). A Wilcoxon test was calculated to check for any significant differences between the first two weeks and the last two weeks. When taking the Bonferroni-correction into account, none of the pairs showed a significant difference, although the case of drug intake was close to significant with $p=.01$. In the last two weeks, the patients recorded manually their blood glucose tests less close to the prescribed intervals than in the first two weeks. Compliance of T2DM patients on drugs decreased in the last two weeks with respect to the compliance of the first two. The adherence to physical activity prescriptions and food prescriptions (in the latter case concerning the number of meals entered) improved slightly. The intake of calories did not change over the course of the trial. Mean compliance was $65 \%$.

Regarding the Control Panel, TP used the tool differently depending on the type of patient. For instance, this manifested itself in the manner professionals visualized the trends of patients clinical data: T1DM patients were supervised 

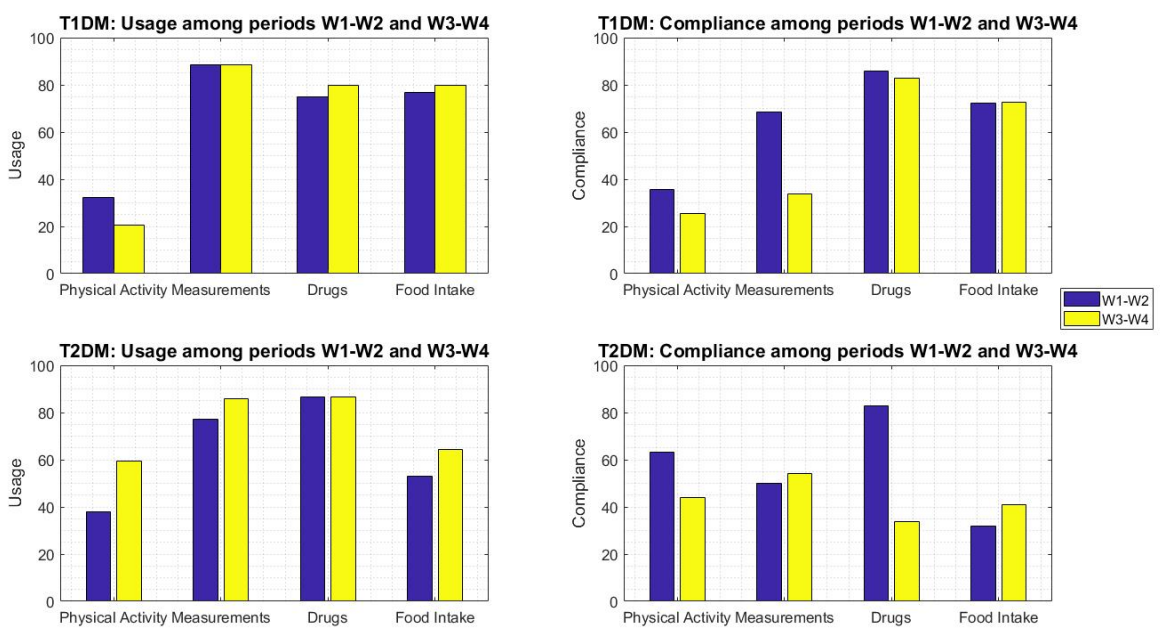

Fig. 6 Usage and Compliance metrics for each module compared during W1-W2 and W3W4 periods for each type of patient

based on complex charts (meal-oriented charts, blood sugar progression after meal charts, etc.) in $78 \%$ of the times. When monitoring T2DM patients, the professionals relied less on the combination of complex charts $(40 \%)$ than on simple charts $(60 \%)$.

\subsection{System attractiveness and perceived usefulness}

The efficacy and the efficiency of both T1DM and T2DM applications was measured using the AttrakDiff, which allows measuring the level of easinessto-use interface. The user experience results for T1DM application are depicted in Fig. 7.

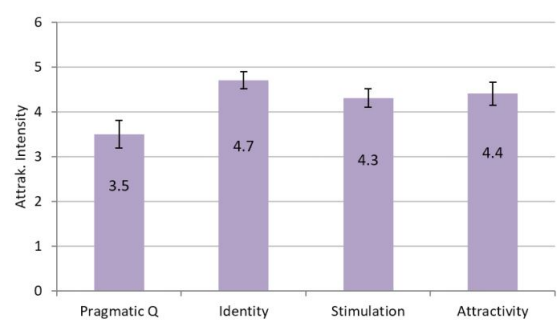

(a) T1DM

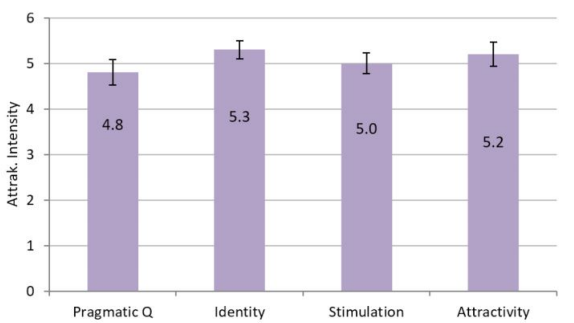

(b) T2DM

Fig. 7 AttrakDiff results per category 
In the case of T1DM, the confidence interval of the pragmatic quality measure overlaps the middle point of the scale, even though the mean value is situated above this critical level. Thus, our quality criterion is missed, even though by the least extent possible. As the other values are far above the middle, one may assume that the users saw the application very positively. A hypothetical explanation could be that the T1DM participants were very used to smartphones and might have considered the windows mobile platform as inferior to iOS or Android based systems. Results from the Perceived Usefulness questionnaire were collected to assess the usefulness of the overall system within the diabetes treatment. Results show a general good level of the patient perceived usefulness $($ mean $=4.60 \% ; \mathrm{SD}=0.9)$.

\section{Discussion}

We designed a system that supports the health management of diabetic patients in their living environments. The frequency of use and the information sent from the app remained similar among T1DM and T2DM patients; however T1DM patients were more likely to use less features of the app. The PERSONAs definition supported the differentiation of user groups that finally resulted in tailored PHR registries. Placing the patient at the centre of the development process through UCD was the key.

Usage statistics show that the patients used the METABO system as much as expected, even though both usage and compliance levels were not excellent, they were acceptably high for such a new system. Both, patients and medical doctors, learned to use the system over time, while increasing their efficiency. T2DM users entered as many measurement values as T1DM ones. Initially, the T2DM PERSONA had been defined as an adult (over 60 years old) with a low level of IT literacy compared to the T1DM PERSONA. Therefore, we thought that T2DM PERSONA could be less capable and even reluctant to use the PMD for the self-management of the disease. We did not collect measures of IT literacy during the sampling procedure (Table 1). Results reject our initial hypothesis, as T2DM participants achieved a comparable level of use and communication metrics, and the differences between the first and the second half of the study on these indicators are smaller than the differences observed in the T1DM group.

Access to and communications from the modules in the mobile applications had different trends, but show statistically independent behaviours (Table 2 and Table 3). Overall, the patients' compliance (Equation 2) related to the modules decreased, but we observed a correlation in T1DM patients regarding the drug prescription and also the food intake. The general compliance showed dramatic changes among the second half of the study. A possible explanation is that after the first two weeks of use, patients started to change their treatments to optimize the health outcomes. Fig. 6 shows how the usage of the modules remained at similar levels, whereas the compliance was significantly reduced. The assessment of such indicators could be an interesting break-point to de- 
termine whether a patient is showing adherence to treatments irrespectively the short-term clinical outcome evolution. Stratification of patients according to their IT literacy and performance on communications, access and use objective metrics could lead to a better performance in terms of personalized medicine [31].

Some features of the METABO system have been previously implemented in diabetes management systems. Nevertheless, these implementations lacked the necessary tailoring of electronic tools to the specific needs of each PERSONA. In our research, we have tried to identify the benefits of user centered design in order to design, deliver and test a tailored solution for diabetes management. Furthermore, the integration of standard sensors for bio-signal acquisition is an essential element in order to implement a reliable information workflow, as confirmed by [32]. The designed system fills the gap identified in a recent review on T2DM mobile apps [13]: from the 89 apps analysed, a majority was of high quality with respect to a single dimension of the disease but only 4 out of 89 apps integrated all six dimensions, and less than half integrated at least four of them.

Even though our study had a limited duration, we were able to determine significant differences in patient response with respect to therapeutic and lifestyle prescriptions. The observation period of two cycles of two weeks each was too short to assume any life-style changes, and moreover prevented us of thinking that a behaviour change may be sustained in the long run. We consider that a longer duration of the study would not have yielded any different findings in the observed metrics, and the differences on the use and communication values would continue as the trends observed in week 4 . Patients play a critical role in choices of lifestyles by, for example, exercising, eating well, and learning about their diabetes and as Quinn and colleagues concluded in the WellDoc Study, some medication intensification strategies may be not required if the patient shows lifestyle pattern shift [18]. The system presented in this study would be capable of reducing the morbidities and the appearance of critical events, such as sudden hypoglycaemia and hyperglycaemia by balancing the drug therapy in combination with diet, physical exercise and learning aspects of the patients, but a larger clinical evaluation is needed to confirm these hypothesis. Further investigation needs to be done correlating the technical results with the clinical and usability outcomes for longer periods of usage.

\section{Conclusion}

User Centered Design enabled us to design and implement a customized system for T1DM and T2DM diabetes management and support. This methodology empowered patients to make their own decisions, choices and helped to expand the concept of Personal Health Record as an addition to the Electronic Health Record. Our analysis shows that after an initial period, T1DM patients were more likely to use less features of the designed system, however the commu- 
nications sent from the mobile application stood similar in T1DM and T2DM patients. This indicates that less use is not associated with a low compliance or adherence.

\section{Funding}

This study was funded by European Commission under the 7th Framework Program grant agreement number 216270.3 .

\section{Conflict of interest}

The authors declare that they have no conflict of interest.

\section{Ethical approval}

All procedures performed in studies involving human participants were in accordance with the ethical standards of the institutional and national research committee and with the 1964 Helsinki declaration and its later amendments or comparable ethical standards.

\section{Informed consent}

Informed consent was obtained from all individual participants included in the study.

\section{References}

1. Ellen Nolte and Martin McKee. Caring for People with Chronic Conditions: A Health System Perspective. McGraw-Hill Education (UK), sep 2008. ISBN 9780335236909.

2. Bodenheimer T, Lorig K, Holman H, and Grumbach K. PAtient selfmanagement of chronic disease in primary care. JAMA, 288(19):24692475, nov 2002. ISSN 0098-7484. doi: 10.1001/jama.288.19.2469. URL http://dx.doi.org/10.1001/jama.288.19.2469.

3. American Diabetes Association. Standards of Medical Care in Diabetes2008. Diabetes Care, 31(Supplement 1):S12-S54, jan 2008. ISSN 0149-5992, 1935-5548. doi: $10.2337 / \mathrm{dc} 08-S 012$. URL http://care.diabetesjournals.org/content/31/Supplement_1/S12.

4. Silvio E. Inzucchi, Richard M. Bergenstal, John B. Buse, Michaela Diamant, Ele Ferrannini, Michael Nauck, Anne L. Peters, Apostolos Tsapas, Richard Wender, and David R. Matthews. Management of Hyperglycemia 
in Type 2 Diabetes, 2015: A Patient-Centered Approach: Update to a position statement of the American diabetes association and the European association for the study of diabetes. Diabetes Care, 38(1):140-149, 2015. ISSN 19355548. doi: 10.2337/dc14-2441.

5. Konstantia Zarkogianni, Eleni Litsa, Konstantinos Mitsis, Po Wu, Chanchala Kaddi, Chihwen Cheng, May Wang, and Konstantina Nikita. A Review of Emerging Technologies for the Management of Diabetes Mellitus. IEEE transactions on bio-medical engineering, $\operatorname{PP}(99)$ : 1, 2015. ISSN 1558-2531. doi: 10.1109/TBME.2015.2470521. URL http://www.ncbi.nlm.nih.gov/pubmed/26292334.

6. Anne T. Reutens, Richard Hutchinson, Ta Van Binh, Clive Cockram, Chaicharn Deerochanawong, Low Tone Ho, Linong Ji, Bin Abdul Kadir Khalid, Alice Pik Shan Kong, Mary Ann Lim-Abrahan, Chee Eng Tan, Askandar Tjokroprawiro, Kun Ho Yoon, Paul Z. Zimmet, and Jonathan E. Shaw. The GIANT study, a cluster-randomised controlled trial of efficacy of education of doctors about type 2 diabetes mellitus management guidelines in primary care practice. Diabetes Research and Clinical Practice, 98 (1):38-45, 2012. ISSN 01688227. doi: 10.1016/j.diabres.2012.06.002. URL http://dx.doi.org/10.1016/j.diabres.2012.06.002.

7. S. Aslan, G. Ciocca, and R. Schettini. Semantic segmentation of food images for automatic dietary monitoring. In 2018 26th Signal Processing and Communications Applications Conference (SIU), pages 1-4, May 2018. doi: 10.1109/SIU.2018.8404824.

8. Enrique J. Gómez, M. Elena Hernando Pérez, Thomas Vering, Mercedes Rigla Cros, Oliver Bott, Gema García-Sáez, Peter Pretschner, Eulalia Bruguéz, Oliver Schnell, Caroline Patte, Joachim Bergmann, Ralf Dudde, and Alberto de Leiva. The INCA system: A further step towards a telemedical artificial pancreas. IEEE Transactions on Information Technology in Biomedicine, 12(4):470-479, 2008. ISSN 10897771. doi: 10.1109/TITB.2007.902162.

9. A Martinez-Millana, G Fico, C Fernández-Llatas, and V Traver. Performance assessment of a closed-loop system for diabetes management. Med Biol Eng Comput, 53(12):1295-1303, dec 2015. ISSN 1741-0444. doi: 10.1007/s11517-015-1245-3.

10. Nicolas Michel Oreskovic, Jennifer Maniates, Jeffrey Weilburg, and Garry Choy. Optimizing the Use of Electronic Health Records to Identify HighRisk Psychosocial Determinants of Health. JMIR Medical Informatics, 5(3):e25, 2017. ISSN 2291-9694. doi: 10.2196/medinform.8240. URL http://medinform.jmir.org/2017/3/e25/.

11. R. Conte, F. Sansone, A. Grande, A. Tonacci, F. Napoli, A. P. Pala, M. Raciti, and P. Landi. Development of an integrated ict system for data production, standardization and elaboration in health amp;amp; care. In 2017 E-Health and Bioengineering Conference (EHB), pages 321-324, June 2017. doi: 10.1109/EHB.2017.7995426.

12. Borim Ryu, Nari Kim, Eunyoung Heo, Sooyoung Yoo, Keehyuck Lee, Hee Hwang, Jeong-Whun Kim, Yoojung Kim, Joongseek Lee, and Se Young 
Jung. Impact of an Electronic Health Record-Integrated Personal Health Record on Patient Participation in Health Care: Development and Randomized Controlled Trial of MyHealthKeeper. Journal of Medical Internet Research, 19(12):e401, 2017. ISSN 1438-8871. doi: 10.2196/jmir.8867. URL http://www.jmir.org/2017/12/e401/.

13. Sarah Chavez, David Fedele, Yi Guo, Angelina Bernier, Megan Smith, Jennifer Warnick, and François Modave. Mobile Apps for the Management of Diabetes. Diabetes Care, 40(10):e145e146, 2017. ISSN 0149-5992. doi: 10.2337/dc17-0853. URL http://care.diabetesjournals .org/lookup/doi/10.2337/dc17-0853.

14. Concetta Irace, Matthias Axel Schweitzer, Cesare Tripolino, Faustina Barbara Scavelli, and Agostino Gnasso. Diabetes Data Management System to Improve Glycemic Control in People With Type 1 Diabetes: Prospective Cohort Study. JMIR mHealth and uHealth, 5 (11):e170, 2017. ISSN 2291-5222. doi: 10.2196/mhealth.8532. URL http://mhealth.jmir.org/2017/11/e170/.

15. Abdelsalam Helal, Diane J Cook, and Mark Schmalz. Smart Home-Based Health Platform for Behavioral Monitoring and Alteration of Diabetes Patients. J Diabetes Sci Technol, 3(1):141-148, jan 2009. ISSN 1932-2968. URL http://www.ncbi.nlm.nih.gov/pmc/articles/PMC2769843/.

16. J Synnott, L Chen, C D Nugent, and G Moore. Flexible and customizable visualization of data generated within Intelligent Environments. In 2012 Annual International Conference of the IEEE Engineering in Medicine and Biology Society, pages 5819-5822, aug 2012. doi: 10.1109/EMBC.2012.6347317.

17. Yuval Shahar, Dina Goren-Bar, David Boaz, and Gil Tahan. Distributed, intelligent, interactive visualization and exploration of time-oriented clinical data and their abstractions. Artif Intell Med, 38(2):115-135, oct 2006. ISSN 0933-3657. doi: 10.1016/j.artmed.2005.03.001.

18. G. Fico, A. Fioravanti, M. Teresa Arredondo, J. Gorman, C. Diazzi, G. Arcuri, C. Conti, and G. Pirini. Integration of personalized healthcare pathways in an ict platform for diabetes managements: A small-scale exploratory study. IEEE Journal of Biomedical and Health Informatics, 20(1):29-38, Jan 2016. ISSN 2168-2194. doi: 10.1109/JBHI.2014.2367863.

19. Salzburg Global Seminar. Salzburg statement on shared decision making. $\quad B M J, \quad 342: \mathrm{d} 1745, \quad$ mar 2011. ISSN 0959-8138, 1468-5833. doi: 10.1136/bmj.d1745. URL http://www . bmj . com/content/342/bmj . d1745.

20. Dawn Stacey, Carol L Bennett, Michael J Barry, Nananda F Col, Karen B Eden, Margaret Holmes-Rovner, Hilary Llewellyn-Thomas, Anne Lyddiatt, France Légaré, and Richard Thomson. Decision aids for people facing health treatment or screening decisions. Cochrane Database Syst Rev, (10):CD001431, 2011. ISSN 1469-493X. doi: 10.1002/14651858.CD001431.pub3.

21. Rose Gabert, Blake Thomson, Emmanuela Gakidou, and Gregory Roth. Identifying High-Risk Neighborhoods Using Electronic Medical Records: A 
Population-Based Approach for Targeting Diabetes Prevention and Treatment Interventions. PloS one, 11(7):e0159227, 2016. ISSN 19326203. doi: 10.1371/journal.pone.0159227.

22. Michael J Barry and Susan Edgman-Levitan. Shared Decision Making The Pinnacle of Patient-Centered Care. New England Journal of Medicine, 366(9):780-781, mar 2012. ISSN 0028-4793. doi: 10.1056/NEJMp1109283. URL http://dx.doi.org/10.1056/NEJMp1109283.

23. Giuseppe Fico, Jorge Cancela, Maria Teresa Arredondo, Arianna Dagliati, Lucia Sacchi, Daniele Segagni, Antonio Martinez Millana, Carlos Fernandez-Llatas, Vicente Traver, Francesco Sambo, et al. User requirements for incorporating diabetes modeling techniques in disease management tools. In 6th European Conference of the International Federation for Medical and Biological Engineering, pages 992-995. Springer, 2015.

24. Boris Draznin, Janice Gilden, Sherita H. Golden, and Silvio E. Inzucchi. Pathways to quality inpatient management of hyperglycemia and diabetes: A call to action. Diabetes Care, 36(7):1807-1814, 2013. ISSN 01495992. doi: $10.2337 / \mathrm{dc} 12-2508$.

25. Jakob Nielsen and Rolf Molich. Heuristic Evaluation of User Interfaces. In Proceedings of the SIGCHI Conference on Human Factors in Computing Systems, CHI '90, pages 249-256, New York, NY, USA, 1990. ACM. ISBN 9780201509328. doi: 10.1145/97243.97281. URL http://doi.acm.org/10.1145/97243.97281.

26. Alejandro Enrique Flores, D Ph, and Victor Medel Vergara. Functionalities of Open Electronic Health Records System. Biomedical Engineering and, (Bmei):602-607, 2013. URL http://ieeexplore. ieee.org/xpls/abs_all.jsp?arnumber $=6747011$.

27. Alejandra Guillén, Javier Colás, Giuseppe Fico, and Sergio Guillén. Metabo: A new paradigm towards diabetes disease management. an innovative business model. In Engineering in Medicine and Biology Society, EMBC, 2011 Annual International Conference of the IEEE, pages 35543557. IEEE, 2011.

28. Marc Hassenzahl, Michael Burmester, and Franz Koller. Attrakdiff: Ein fragebogen zur messung wahrgenommener hedonischer und pragmatischer qualität. In Mensch \& Computer 2003, pages 187-196. Springer, 2003.

29. Fred D Davis. Perceived usefulness, perceived ease of use, and user acceptance of information technology. MIS quarterly, pages 319-340, 1989.

30. Alessio Fioravanti, Giuseppe Fico, Dario Salvi, Rebeca I GarcaBetances, and Maria Teresa Arredondo. Automatic messaging for improving patient's engagement in diabetes management: an exploratory study. Med Biol Eng Comput, 53(12):12851294, December 2015. ISSN 0140-0118. doi: 10.1007/s11517-014-1237-8. URL https://doi.org/10.1007/s11517-014-1237-8.

31. Linda Haas, Melinda Maryniuk, Joni Beck, Carla E. Cox, Paulina Duker, Laura Edwards, Edwin B. Fisher, Lenita Hanson, Daniel Kent, Leslie Kolb, Sue McLaughlin, Eric Orzeck, John D. Piette, Andrew S. Rhinehart, Russell Rothman, Sara Sklaroff, Donna Tomky, and Gretchen Youssef. 
National standards for diabetes self-management education and support. Diabetes Care, 35(11):2393-2401, 2012. ISSN 01495992. doi: 10.2337/dc121707.

32. Charlene C. Quinn, Patricia L. Sareh, Michelle L. Shardell, Michael L. Terrin, Erik A. Barr, and Ann L. Gruber-Baldini. Mobile Diabetes Intervention for Glycemic Control. Journal of Diabetes Science and Technology, 8 (2):362-370, 2014. ISSN 1932-2968. doi: 10.1177/1932296813514503. URL http://journals.sagepub.com/doi/10.1177/1932296813514503. 